\title{
Análise dos indicadores de inovação tecnológica no Brasil: comparação entre um grupo de empresas privatizadas e o grupo geral de empresas*
}

\author{
Elisa Maria Pinto da Rocha \\ Economista, estudante de doutorado em ciência da informação/ \\ UFMG; professora titular do Unicentro Newton Paiva e \\ pesquisadora da Fundação João Pinheiro. \\ E-mail: lirocha@horizontes.net
}

\author{
Marta Araújo Tavares Ferreira \\ Professora do Programa de Pós-Graduação em Ciência da Informação \\ da Universidade Federal de Minas Gerais (PPGCI-UFMG). \\ E-mail:maraujo@eb.ufmg.br
}

\section{INTRODUÇÃO}

Na última década, observa-se mudança da dinâmica tecnológica mundial, cuja manifestação mais explícita relaciona-se à ocorrência de grande número de inovações. A aplicação do novo conhecimento a situações empíricas redundou no rápido avanço tecnológico, tornando o processo produtivo cada vez mais complexo, em uma dinâmica auto-alimentadora, na qual as mudanças tecnológicas decorrentes de inovações em produtos e processos engendram necessidades que, por sua vez, alimentam outras soluções, produtos e processos, em uma dinâmica continuada e interativa.

O processo de inovação tecnológica é, entretanto, apenas uma das faces de um fenômeno bem mais amplo e profundo vivido pelas sociedades contemporâneas: a mudança do próprio paradigma sociocultural associada a uma transformação do padrão de acumulação capitalista. Neste novo cenário da Sociedade do Conhecimento, informação e conhecimento ganham renovado destaque e passam a se constituir objeto de estudo privilegiado de áreas como a das ciências econômicas e administrativas, ciências da computação e da comunicação, sociologia, pedagogia e antropologia, entre outras. Os aspectos mais fundamentais relacionados à mudança do paradigma é o significado deste fenômeno. Segundo o autor, o que distingue o momento atual dos anteriores é o sociocultural e sua interface. E possíveis impactos na socidade têm sido retratados na literatura da ciência da informação. Além disso, dentre as abordagens que discutem o alcance e a natureza das transformações da sociedade contemporânea, as análises de Giddens (1991) ilustram a magnitude e ritmo e a abrangência das mudanças na natureza das instituições modernas, em que o avanço das tecnologias da comunicação e o desenvolvimento dos sistemas peritos de organização da vida (discursos competentes) resultaram em uma separação do tempo e do espaço. Por sua vez, as questões sobre a dimensão informacional e a

\footnotetext{
* Este artigo reflete algumas das discussões apresentadas no estudo "Uso dos indicadores de inovação como fonte de informação sobre o desempenho tecnológico: considerações sobre o caso das empresas brasileiras privatizadas", elaborado pelas autoras no âmbito do Programa de Pós-Graduação em Ciência da Informação da Universidade Federal de Minas Gerais (PPGCI-UFMG).
} 
nova infra-estrutura necessária ao crescente fluxo da informação têm resultado no desenvolvimento de novas designações e conceitos, a exemplo do "espaço dos fluxos", de Castells (1996), e do "ciberespaço", de Levy (1997).

As habilidades de se gerar e compartilhar informação e conhecimento e informação são, atualmente, consideradas vetores fundamentais para a determinação das vantagens competitivas de países e empresas, influindo no crescimento econômico, na geração de riqueza e na melhoria da qualidade de vida de uma sociedade. Esta percepção se reflete nas próprias experiências mais recentes conduzidas por empresas, institutos e centros de pesquisa públicos e privados localizados nos países desenvolvidos.

$\mathrm{Na}$ Organização de Cooperação para o Desenvolvimento Econômico (OCDE), por exemplo, a concepção de que o progresso da ciência e a mudança tecnológica, propiciados pela interação entre conhecimento e informação, constitui vetor-chave das transformações ora em curso e tem motivado a elaboração de uma vasta gama de estudos e pesquisas, com o objetivo de aprofundar a compreensão sobre a natureza do processo inovador dos países membros, no contexto da nova "economia baseada no conhecimento" (knowledge-based economy). Recentes iniciativas da OCDE, como a implementação do projeto "Blue Sky" e do programa de estudos e pesquisas "Reestructuring and reintegration of SETT systems in economies in transition" (1998) e o aperfeiçoamento da metodologia contida no Manual de Oslo (versão original de 1992 e primeira revisão de 1996), representam ações concretas dos países desenvolvidos no sentido de ampliar o escopo das "políticas de ciência e tecnologia" e de adequar a configuração dos atuais sistemas de informações em C\&T, ajustando-os aos requerimentos impostos pela sociedade do conhecimento. A crescente preocupação dos países centrais em compreender a natureza e a complexidade dos processos de inovação tecnológica tem sido acompanhada por uma mudança de foco nas discussões sobre as transformações ocorridas na base tecnoeconômica das sociedades contemporâneas. As abordagens baseadas nas "políticas de ciência e tecnologia" estão sendo gradativamente substituídas pela concepção dos "sistemas nacionais de inovação", tendência que se manifesta, principalmente, a partir dos trabalhos pioneiros de Lundvall (1992) e Nelson (1993) sobre "sistemas nacionais de inovação".

O processo de inovação tecnológica passa a dominar as discussões sobre estratégia de inserção internacional de empresas e países, pela importância que vem assumindo enquanto fator determinante da competitividade e mesmo da sobrevivência das empresas. No Brasil, entretanto, o assunto não tem merecido a atenção que recebe nas economias desenvolvidas. E, no caso específico das empresas brasileiras privatizadas, é imensa a carência de informações sobre o comportamento tecnológico das "exestatais". As pesquisas sobre as empresas privatizadas focalizam apenas os aspectos negociais e contábeis, não fornecendo, portanto, quaisquer informações sobre o impacto das privatizações no processo de inovação tecnológica do país, e esta é, justamente, a idéia central que norteou a elaboração da pesquisa conduzida no âmbito do Programa de Pós-Graduação em Ciência da Informação da Universidade Federal de Minas Gerais (PPGCIUFMG).

Os formuladores da política econômica brasileira esperavam que a venda das estatais assegurasse maior dinamismo às empresas, o que contribuiria positivamente para o desenvolvimento tecnológico do país como um todo. Passados quase três anos do início das privatizações, algumas questões carecem de aprofundamento:

- As empresas privatizadas estariam, de fato, realizando um maior esforço inovador?

- Existiriam grandes diferenças e/ou semelhanças entre o processo de inovação tecnológica das empresas privatizadas e das demais empresas brasileiras?

- Qual a trajetória do emprego nestas empresas que se caracterizavam pelo uso mais intensivo de mão-de-obra qualificada?

\section{CARACTERIZAÇÃO DAS AMOSTRAS DE EMPRESAS}

Os dados básicos utilizados na pesquisa foram extraídos da base de dados de indicadores empresariais de inovação tecnológica da Anpei, que compreende cerca de 23 indicadores que procuram retratar algumas dimensões do processo de inovação tecnológica das empresas, tais como intensidade dos esforços de inovação, atividades inovadoras e resultados dos investimentos em inovação sobre a empresa (em termos de receita e de custos).

Para efeitos da pesquisa, foram analisados os indicadores que mostraram maior consistência ao longo da série 1994 a 1998. Os indicadores Anpei se referem a valores médios por empresa de cada grupo analisado e, neste sentido, como qualquer outro indicador, devem ser tomados como "sinais" tendenciais de comportamento, e não como medidas exatas das variáveis que expressam. 
Foram considerados dois grupos de empresas da base Anpei ${ }^{1}$ :

a) Grupo de Empresas Privatizadas: composto por cerca de 10 empresas selecionadas a partir do ano de privatização, de acordo com publicações do BNDES (1999); o faturamento do grupo corresponde a cerca de $4 \%$ do produto interno bruto do setor industrial (PIB industrial de 1998).

Os indicadores originalmente expressos em valores (dólares correntes) foram deflacionados pelo IPC norteamericano, ano-base de 1998, e transformados em dólar pelo valor médio daquele ano.

b) Grupo Geral de Empresas: formado por todas as empresas da base, cerca de 400 empresas, cujo faturamento corresponde a cerca de $30 \%$ do PIB industrial do país.

A análise dos principais indicadores - intensidade do esforço inovador, disponibilidade de recursos humanos qualificados para as atividades inovadoras, patentes resultados dos esforço de inovação sobre a receita e os custos da empresa - é feita a seguir.

\section{ANÁLISE DO DESEMPENHO INOVADOR DOS GRUPOS DE EMPRESAS}

O investimento total das empresas em inovação tecnológica corresponde aos gastos realizados em um conjunto de atividades inovadoras, com o objetivo de aumentar o estoque e a aplicabilidade do conhecimento tecnológico, bem como elevar o nível das competências e habilidades dos recursos produtivos da empresa. Além do núcleo central, relacionado à aplicação de recursos financeiros nas atividades de "pesquisa e desenvolvimento" (P\&D), os investimentos em inovação tecnológica das empresas compreendem os dispêndios com "serviços de apoio tecnológico" (capacitação de recursos humanos alocados nas atividades de pesquisa e desenvolvimento, ensaios, testes, análises técnicas e informações tecnológicas), com a "aquisição de tecnologia" (royalties pagos pelo uso de marcas e patentes, aquisição de direitos relacionados com novos produtos ou processos, serviços de assistência técnica, aquisição de programas de computador), os gastos com "engenharia não rotineira" (design, rearranjos de plantas requeridas

\footnotetext{
${ }^{1}$ A Anpei representa hoje, no caso brasileiro, a única fonte sistematizada de informações sobre os investimentos em inovação realizados pelas empresas do país e, por questões de sigilo e de segurança das empresas informantes, disponibiliza seus indicadores para um conjunto mínimo de cinco empresas.
}

FIGURA 1

Esforço inovador segundo grupos de empresas: 1994. $1998^{*}$

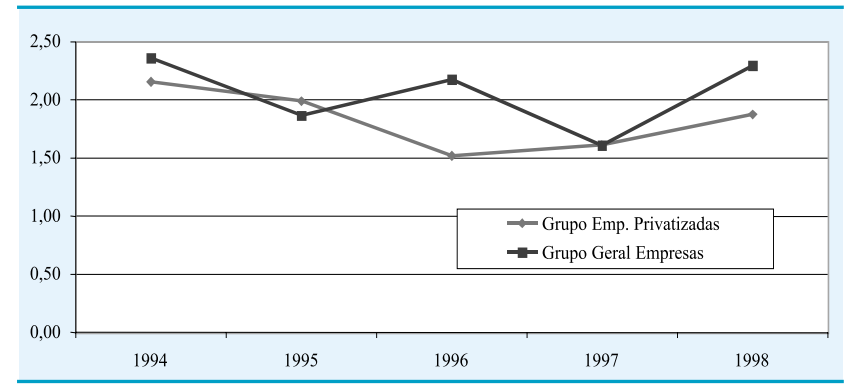

Fonte: dados básicos da Anpei/nov.1999, compilados pelas autoras. Obs.: referem-se a valores médios das empresas de cada grupo.

* Investimento total em inovação tecnológica como percentual do faturamento bruto.

para implementação de novos produtos e processos), os "investimentos físicos" (compra de máquinas, equipamentos e instalações) e os "investimentos intangíveis" (direitos sobre licenças para exploração de patentes e uso de marcas e contratos de fornecimento de tecnologia industrial), segundo a Anpei (1998).

Embora, em valores absolutos, tenha sido registrado aumento dos investimentos em inovação tecnológica para os dois grupos de empresas, este crescimento não tem sido proporcional à elevação do faturamento bruto das empresas (figura 1). Em decorrência, registra-se uma tendência de queda no indicador do esforço inovador relativo ao Grupo de Empresas Privatizadas (em 1994, as empresas aplicaram, em média, 2,2\% do seu faturamento em inovação; em 1998, este percentual caiu para 1,9\%). No caso do Grupo Geral de Empresas, o índice se manteve praticamente inalterado (percentuais de 2,4\%, em 1994, e de 2,3\%, em 1998). As oscilações deste indicador durante o período 1994-1998 registradas para os dois grupos de empresas indicam que os planos empresariais de investimentos em inovação são bastante influenciados pelos movimentos conjunturais da economia. Portanto, no Brasil, são ainda frágeis as estratégias das empresas no que se refere a investimentos que envolvem maiores incertezas e prazos de maturação mais longos, como é o caso dos investimentos em inovação.

No que se refere às atividades inovadoras, registra-se queda da importância relativa dos gastos com "P\&D" para o Grupo de Empresas Privatizadas (em 1994 estes gastos correspondiam a $68,4 \%$ do investimento total em inovação, tendo crescido para 65,0\% em 1998), simultaneamente a um vertiginoso crescimento da participação relativa da categoria "aquisição de tecnologia" (este item que representava 5,2\% do investimento total 
em inovação no primeiro ano da série, elevou-se para 13,1\% em 1998) (ver quadro $1 \mathrm{~A}$-Apêndice). Este resultado sugere uma tendência de aumento da compra de tecnologia (sob a forma de pagamentos de assistência técnica e de uso de marcas e patentes), em detrimento dos esforços de geração autônoma por parte das empresas privatizadas.

No novo contexto da economia baseada no conhecimento, a existência de um quadro de pessoal qualificado tende a potencializar o desenvolvimento tecnológico, e, a este respeito, os resultados da pesquisa indicam uma situação preocupante. De acordo com os dados mostrados no quadro 2A do Apêndice, observa-se, no caso de ambos os grupos de empresas, que apenas uma pequena parcela (menos de 15\%) dos técnicos envolvidos com as atividades inovadoras possui qualificação compatível com as habilidades requeridas pelo processo de inovacão. Para o ano de 1998, a participação de mestres e doutores no total dos técnicos envolvidos com atividades inovativas foi, em média, $12,1 \%$ no caso do Grupo de Empresas Privatizadas, e 14,4\% para o Grupo Geral de Empresas. Assim, os pesquisadores mais qualificados não estão atuando nas empresas, mas, principalmente, em universidades e institutos de pesquisa.

Além disso, chama atenção a forte queda (da ordem de 42\% no período 1994 a 1998) do número de profissionais com nível superior referente ao Grupo de Empresas Privatizadas, não tendo sido poupados nem mesmo aqueles os funcionários com maior nível de qualificação (mestres e doutores). No caso do Grupo Geral de Empresas, a retração se mostrou bem mais amena $(1,8 \%$ na mesma base de comparação) ${ }^{2}$.

As estatísticas de patentes têm sido cada vez mais utilizadas como proxies do resultado da pesquisa tecnológica, em que pesem as limitações que envolvem esta aproximação, sobretudo em países como o Brasil, onde a prática do registro de marcas e patentes é pouco generalizada. De qualquer forma, conforme ressaltado por Patel \& Pavitt (1998), a carência de indicadores mais adequados, capazes de mensurar a totalidade dos aspectos relativos à produção de tecnologia, justifica o uso destes indicadores, cuja disseminação entre diversos países do mundo tem, inclusive, reforçado as iniciativas governamentais no sentido de aperfeiçoar as bases de dados sobre patentes, especialmente nos países da OCDE.

\footnotetext{
${ }^{2}$ Estes resultados são compatíveis com as conclusões de Biondi (1999) quanto aos impactos negativos das privatizações sobre o nível de emprego na economia brasileira.
}

FIGURA 2

\section{Portfólio de patentes, segundo grupos de empresas: 1994 - 1998*}

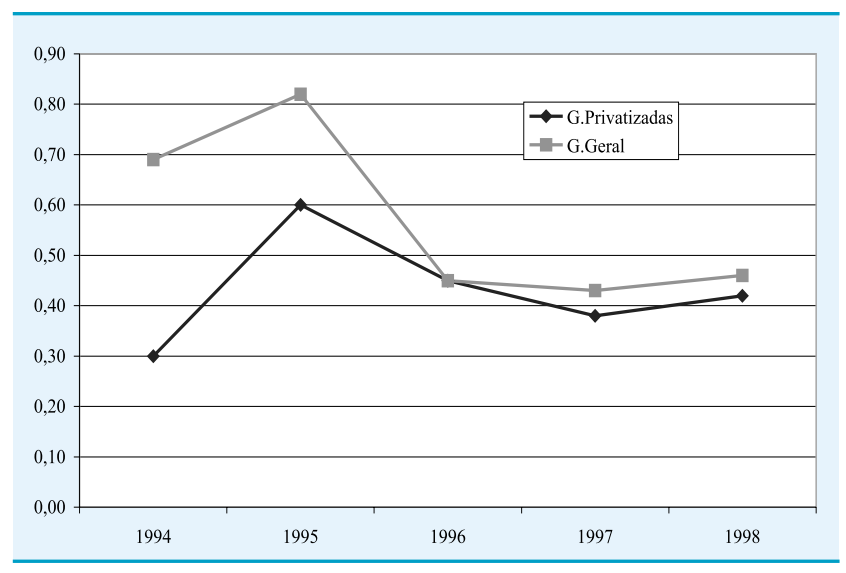

Fonte: dados básicos da Anpei/nov.1999, compilados pelas autoras.

* Valores médios por empresa de cada grupo relativos às patentes concedidas e/ou depositadas (média anual dos últimos 10 anos).

Conforme mostrado na figura 2, a comparação deste indicador entre os dois grupos de empresas indica que, durante todo o período analisado, o número de patentes concedidas e/ou depositadas no Brasil relativo ao grupo de empresas privatizadas mostrou-se menor do que aquele registrado pelo grupo geral de empresas da base Anpei. Entretanto, mais interessante do que a evolução do número absoluto de patentes de cada grupo foi o movimento de queda registrado a partir de 1995 - período de crise, após início do Plano Real, que atingiu ambos os grupos de empresas. Desde aquele ano, observa-se uma situação geral de estagnação.

A inovação tecnológica tende a gerar efeitos positivos sobre a receita (em função da venda de novos produtos) e sobre os lucros da empresa (em função da economia de custos decorrente de novos processos e métodos produtivos introduzidos pelas empresas). Também nestes casos, os indicadores de impactos apontaram uma situação desconfortável para o grupo de empresas privatizadas. Em 1998, por exemplo, a comercialização de novos produtos por parte das firmas deste grupo respondeu por apenas 8,0\% do faturamento médio das empresas, percentual bem menor do que o registrado para o grupo geral, cerca de $36,4 \%$. No que se refere à economia de custos operacionais, representou $0,54 \%$ do lucro bruto médio das firmas do grupo empresas privatizadas, contra um percentual de $1,2 \%$ para o grupo geral. Estes resultados relativamente mais desfavoráveis ao grupo de privatizadas é, entretanto, compatível com o menor esforço inovador registrado para este grupo e refletido na menor proporção do faturamento direcionado aos investimentos em inovação. 


\section{CONCLUSÕES}

Embora as amostras pesquisadas não sejam ideais, os indícios fornecidos pela pesquisa vão contra a idéia geral de que a privatização tenha se constituído um mecanismo suficiente para dinamizar o desenvolvimento tecnológico das empresas. Além disso, é preocupante o fato de as empresas privatizadas manifestarem uma opção mais forte pela compra de tecnologia, em vez de reforçar seus projetos estratégicos de geração autônoma de inovações.

É urgente a necessidade de se debater seriamente a questão da inserção da economia brasileira na sociedade do conhecimento, e, neste campo, diferentemente das posições neoliberais, as evidências colhidas nos países industrialmente avançados indicam que os "estados nacionais" têm importante papel a cumprir: articular ações integradas e redes de P\&D entre empresas, universidades, centros e institutos de pesquisa, de forma a transformar as atuais estruturas de ciência e tecnologia em eficientes sistemas nacionais de inovação. Tal estratégia vai muito além da recente aprovação de fundos setoriais, o que, entretanto, pode representar o início de um longo caminho a ser trilhado.

\section{REFERÊNCIAS BIBLIOGRÁFICAS}

ANPEI. Indicadores empresariais de inovação tecnológica: resultados da base de dados ANPEI. ANPEI, v. 7, dez. 1998. 68 p.

ANPEI. Tabulações especiais realizadas pela ANPEI e cedidas ao Núcleo Gerencial e Tecnológico do Curso de Pós-Graduação em Ciência da Informação da UFMG. [S. l.], 1999.

BIONDI, A. O Brasil privatizado - um balanço do desmonte do Estado. 4. ed. [S. 1.] : Fundação Perseu Abramo, 1999. 48 p.

BNDES. Secretaria Geral de Apoio à Desestatização (Rio de Janeiro). Programa Nacional de Desestatização-Sistema de Informações. [Rio de Janeiro] , 1999.

CASTELLS, M. The information age: economy, society and culture. Oxford : Blackwell, 1996.

GIDDENS, A. As conseqüências da modernidade. In: UNESP (São Paulo). Sistemas abstratos e transformação da intimidade. São Paulo, 1991. cap. 4 , p. $115-150$.

LEVY, P. A. Inteligência coletiva. Lisboa : Instituto Piaget, 1997.

LUNDVALL, B. A. National systems of innovation: towards a theory of innovation and interactive learnig. Londres : Printer Publisher, 1992. $341 \mathrm{p}$.

NELSON, R.R. (Ed.). National Innovation Systems: a comparative analysis. New York : Oxford University, 1993. 541 p.

OCDE. Fostering scientific and technological progress. Observer OECD, June 1999. Disponível em: <http://www.oced.org5/news and events/ publish/pb98-32a.htm>

OCDE. Main science and technology indicators. [S. 1.] , 1999. 83 p. (OCDE, n.1).

OCDE. Manual de Oslo: OECD proposed guidelines for collecting and interpreting technological innovation data. Paris, 1996.

PATEL, P. ; PAVITT, K. National systems of innovation under strain: the internationalisation of corporate R\&D. [S. 1.] : Science Policy Research Unit. University of Sussex, 1998. (Eletronic Working Papers Series, n. 22). Disponível em: <http://www. Sussex.ac.uk/spru>
Agradecimentos
As autoras agradecem a participação da Associação Nacional de Pesquisa
das Empresas Industriais (Anpei), cuja colaboração tornou possível a
realização do estudo, e às estagiárias do Núcleo de Informação Gerencial
e Tecnológica, Denise Silva e Cláudia Souza, que articularam a
disponibilização dos dados e informações necessárias ao trabalho. 
Análise dos indicadores de inovação tecnológica no Brasil

\section{APÊNDICE}

\section{QUADRO 1A}

Mix das atividades inovativas: participação das categorias de gastos no valor do investimento total em inovação tecnológica, segundo grupos de empresas: 1994-1998 (\%)

\begin{tabular}{|c|c|c|c|c|c|c|c|c|c|c|}
\hline \multirow[t]{2}{*}{ Categoria de gasto em inovação tecnológica } & \multicolumn{5}{|c|}{ Grupo de Empresas Privatizadas } & \multicolumn{5}{|c|}{ Grupo Geral de Empresas } \\
\hline & 1994 & 1995 & 1996 & 1997 & 1998 & 1994 & 1995 & 1996 & 1997 & 1998 \\
\hline Pesquisa e Desenvolvimento (P\&D) (1) & 68,4 & 77,4 & 84,9 & 88,8 & 65,0 & 43,8 & 51,8 & 45,9 & 59,2 & 48,7 \\
\hline Serviços Tecnológicos (2) & 24,9 & 3,2 & 3,3 & 7,1 & 17,6 & 11,5 & 10,4 & 7,4 & 12,0 & 9,7 \\
\hline Aquisição de Tecnologia (3) & 5,2 & 15,2 & 6,2 & 4,1 & 13,0 & 8,0 & 7,7 & 6,1 & 9,8 & 8,2 \\
\hline Invest.Físicos & 0,9 & 3,4 & 2,7 & 0,0 & 0,2 & 32,3 & 19,3 & 39,2 & 15,2 & 29,2 \\
\hline
\end{tabular}

FONTE: Anpei, nov./1999, e tabulações das autoras.

Valores em US\$ 1.000, deflacionados pelo Índice Deflator dos PIB dos EUA (base 1998 e US\$ = 1,16).

(1) Agrega a pesquisa básica, a aplicada, o desenvolvimento experimental e a engenharia não rotineira.

(2) Inclui gastos com análises, testes, capacitação, recursos humanos, informações tecnológicas.

(3) Inclui royalties decorrentes de lidenças para uso de marcas e patentes, aquisição de direitos relacionados com novos produtos e processos, bem como de programas de computação.

(4) Inclui máquinas, equipamentos, instalações e terrenos.

(5) Inclui direitos sobre licenças para exploração de patentes e de marcas relacionadas a novos produtos e processos, além de contratos de fornecimento de tecnologia.

\section{QUADRO 2A}

Qualificação do quadro de pessoal alocado em atividades inovadoras, segundo grupo de empresas: 1994-1998.

\begin{tabular}{|c|c|c|c|c|c|c|c|c|c|c|c|c|}
\hline \multirow{3}{*}{ Qualificação do Pessoal } & \multicolumn{6}{|c|}{ Grupo de Empresas Privatizadas } & \multicolumn{6}{|c|}{ Grupo Geral de Empresas } \\
\hline & \multicolumn{5}{|c|}{ Participação (\%) } & \multirow{2}{*}{\begin{tabular}{|l|} 
Txa.Cres.(1) \\
$1994-98(\%)$ \\
\end{tabular}} & \multicolumn{5}{|c|}{ Participaç̃o (\%) } & \multirow{2}{*}{\begin{tabular}{|l} 
Txa.Cres.(1) \\
$1994-98(\%)$ \\
\end{tabular}} \\
\hline & 1994 & 1995 & 1996 & 1997 & 1998 & & 1994 & 1995 & 1996 & 1997 & 1998 & \\
\hline Nível Médio & 35,74 & 21,98 & 17,08 & 14,39 & 17,41 & $-78,19$ & 33,38 & 43,01 & 39,97 & 36,94 & 38,30 & 7,37 \\
\hline Nível Superior & 56,77 & 62,12 & 67,87 & 76,43 & 73,34 & $-42,14$ & 42,14 & 44,90 & 49,25 & 47,64 & 44,25 & $-1,76$ \\
\hline Graduação & 48,13 & 35,05 & 45,21 & 51,56 & 61,24 & $-43,10$ & 34,58 & 31,12 & 35,67 & 36,41 & 29,85 & $-19,23$ \\
\hline Administrativo & 7,49 & 15,90 & 15,05 & 9,18 & 9,25 & $-44,69$ & 24,48 & 12,09 & 10,78 & 15,42 & 17,45 & $-33,3$ \\
\hline Total Pessoal em atividade inov. & 100,00 & 100,00 & 100,00 & 100,00 & 100,00 & $-55,21$ & 100,00 & 100,00 & 100,00 & 100,00 & 100,00 & $-6,4$ \\
\hline
\end{tabular}

FONTE: dados básicos da Anpei, nov./1999. Elaboração das autoras.

(1) Refere-se à taxa de crescimento do pessoal full time, correspondente se refere à variação acumulada no período 1994 a 1998. 